\title{
INTERVENÇÕES FARMACÊUTICAS E SUA IMPORTÂNCIA NA SEGURANÇA DO PACIENTE HOSPITALIZADO
}

\author{
Nayara Aparecida Maioli, Hernani Cesar Barbosa Santos \\ ${ }^{1}$ Universidade do Oeste Paulista - UNOESTE, Residente da Residência multiprofissional em terapia intensiva, \\ Presidente Prudente, SP. Universidade do Oeste Paulista - UNOESTE, Docente Departamento de Farmácia, Presidente \\ Prudente, SP. E-mail: naymaioli@gmail.com
}

\section{RESUMO}

Este trabalho teve como objetivo avaliar as intervenções farmacêuticas realizadas durante a análise das prescrições médicas e a representatividade do profissional farmacêutico na prevenção de erros relacionados a medicamentos. Os dados foram coletados a partir dos registros disponíveis na tabela de Intervenções e Atuações do Farmacêutico Clínico, utilizada na rotina, em um período de 8 meses. Foram analisadas as variáveis: posologia, via de administração, forma farmacêutica, medicamento inapropriado, inconsistências na dose e exames de glicemia desnecessários. Foram notificadas 933 intervenções, sendo 263 referentes à posologia, 139 a via de administração, 47 a forma farmacêutica, 116 a medicamento inapropriado, 310 a dose e 58 a exames de glicemia desnecessários, demonstrando a importância do farmacêutico clínico na prevenção de erros. A segurança do paciente depende da participação ativa do farmacêutico, pois estes contribuíram muito na minimização de danos que poderiam colocar em risco a vida dos pacientes internados.

Palavras-chave: Segurança do paciente, atenção farmacêutica, prescrições de medicamentos, Assistência Farmacêutica, Protocolos Clínicos.

\section{PHARMACEUTICAL INTERVENTIONS AND THEIR IMPORTANCE ON THE SECURITY OF THE HOSPITALIZED PATIENT}

\begin{abstract}
This study aimed to evaluate the pharmaceutical interventions performed during the analysis of medical prescriptions and the representativeness of the pharmaceutical professional in the prevention of medication related errors. The data were collected from the records available in the Clinical Pharmacist Interventions and Actions table, used routinely, over a period of 8 months. The variables were: dosage, route of administration, pharmaceutical form, inappropriate drug, dose inconsistencies and unnecessary blood glucose tests. 933 interventions were reported, 263 referring to the posology, 139 the route of administration, 47 the pharmaceutical form, 116 the inappropriate drug, 310 the dose and 58 to unnecessary blood glucose tests, demonstrating the importance of the clinical pharmacist in the prevention of errors. The safety of the patient depends on the active participation of the pharmacist, as these have contributed greatly in minimizing damages that could endanger the lives of inpatients.
\end{abstract}

Keywords: Patient safety, pharmaceutical care, drug prescriptions, Pharmaceutical Services, Clinical Protocol.

\section{INTRODUÇÃO}

A segurança do paciente tem se tornado preocupação central para o sistema de saúde desde a década de 90. Dados de pesquisa, obtidos dos Estados Unidos, em 1999, indicam que os erros de cuidado à saúde causam, aproximadamente, de 44 a 98 mil eventos adversos por ano nos hospitais daquele país. A partir de então, tem se destacado um movimento global para a segurança do paciente. Em 2004, a 
Organização Mundial de Saúde (OMS) criou o projeto Aliança Mundial para a Segurança do Paciente, cujo objetivo fundamental é prevenir danos aos pacientes hospitalizados ${ }^{1}$.

$\mathrm{O}$ alcance da segurança do paciente tem um único objetivo: a prevenção e melhoria de eventos adversos ou lesões resultantes dos processos de cuidados de saúde. Estes eventos incluem erros, desvios, acidentes e todo tipo de danos desnecessários associados à assistência a saúde. A segurança emerge da interação dos componentes do sistema, que faz não residir em uma única pessoa, dispositivo ou departamento ${ }^{2}$.

Os problemas relacionados aos medicamentos (PRM) são comuns e considerados a principal causa de eventos adversos, sendo que a maioria é evitável e constituem erros de medicação. Estes erros são responsáveis por um aumento significativo no tempo de internação, morbidade, mortalidade e custos hospitalares ${ }^{3}$.

Erros envolvendo medicamentos ocorrem frequentemente em âmbito hospitalar e são classificados como eventos adversos a medicamentos (EAM) podendo ou não causar danos ao paciente ${ }^{4}$. O evento adverso a medicamento abrange a reação adversa a medicamentos (RAM) e o erro de medicação (EM). O EM é um dos mais frequentes tipos de EAM e tem como características o fato de poder ser evitado, ocorrer em qualquer etapa do sistema de medicação (prescrição, dispensação e administração de medicamentos) e com qualquer profissional da equipe multidisciplinar responsável por ações voltadas à terapia medicamentosa: médicos, farmacêuticos e enfermeiros ${ }^{5}$.

Em 2006, um parecer do Colégio Americano de Obstetrícia e Ginecologia (ACOG) sobre o uso seguro de medicamentos, enfatizou que os erros de prescrição é o principal evento adverso a medicamentos. Esses erros envolvem medicamento incorreto, posologia, dose, duração do tratamento e vias de administração. Fatores de ordem técnica também podem contribuir para incidência de erros de prescrição, tais como legibilidade em prescrições manuais, uso de abreviaturas consideradas perigas e descrições inadequadas de dose ${ }^{6}$.

Para que um erro não ocorra, ou caso ocorra, não alcance o paciente, é necessário que haja uma atuação efetiva da equipe multidisciplinar. A atuação do farmacêutico é indispensável para que possa avaliar, detectar e intervir reduzindo possíveis riscos associados à terapêutica medicamentosa. Vários estudos apontam que a avaliação e intervenção farmacêutica reduzem inúmeros erros de prescrição e aumenta a qualidade da farmacoterapia ${ }^{7}$.

As atividades do farmacêutico clínico desempenham papel fundamental na promoção do uso racional de medicamentos, garantindo ao paciente uma farmacoterapia adequada, com resultados terapêuticos definidos e minimizando os riscos de resultados desfavoráveis da terapia medicamentosa, garantindo maior segurança ao paciente ${ }^{8}$.

Este trabalho teve como objetivo analisar as intervenções feitas pela equipe de farmacêuticos clínicos de um hospital público do interior de São Paulo durante a avaliação das prescrições médicas e avaliar a representatividade da atuação do profissional na prevenção dos erros relacionados a medicamentos em busca da segurança do paciente.

\section{METODOLOGIA}

Estudo retrospectivo, no qual foram analisados os registros do setor de farmácia clínica de um hospital universitário, terciário, referentes às intervenções farmacêuticas aceitas durante 0 desenvolvimento das atividades clínicas rotineiras do farmacêutico clínico nos setores de Unidade de Terapia Intensiva, Unidade de Terapia Intensiva Cardiológica, Clínica Médica, Clínica de Especialidades e Cardiologia Clínica, no período de 8 meses, sendo de setembro de 2014 à abril de 2015. O estudo foi aprovado pelo Comitê de Ética em Pesquisa, com a autorização da coordenação da Divisão de Farmácia.

As intervenções farmacêuticas analisadas foram coletadas a partir dos registros farmacêuticos disponíveis na tabela de Intervenções e Atuações do Farmacêutico Clínico, utilizada na rotina do farmacêutico clínico na instituição e elaborada para este fim, utilizando o programa Microsoft Office Excel ${ }^{\circledR}$ 2007. Sobre os registros das intervenções farmacêuticas aceitas, foram analisadas as seguintes variáveis: posologia, via de administração, forma farmacêutica, medicamento inapropriado, inconsistências na dose prescrita e exames de glicemia desnecessários. Os resultados apresentados foram tabulados, organizados e 
apresentados usando estatística descritiva, por meio do Microsoft Office Excel ${ }^{\circledR} 2007$.

\section{RESULTADOS}

Pode-se observar que no período de 8 meses da realização do estudo foram notificadas 933 intervenções farmacêuticas aceitas realizadas por 3 farmacêuticos que revezam os turnos de trabalho. Não houve grandes oscilações das intervenções entre os meses, com um aumento discreto de fevereiro a abril de 2015 , sendo que o mês que mais apresentou intervenções foi o mês de abril. A quantidade de intervenções variou entre 93 e 148 por mês analisado (Tabela 1).

Tabela 1. Distribuição das intervenções farmacêuticas por mês e profissional.

\begin{tabular}{lccc}
\hline Mês & N $^{\circ}$ de intervenções & \% de intervenções & Média por profissional \\
\hline Setembro/2014 & 93 & 9,9 & 31 \\
\hline Outubro/2014 & 115 & 12,3 & 38,3 \\
\hline Novembro/2014 & 111 & 11,9 & 37 \\
\hline Dezembro/2014 & 114 & 12,2 & 38 \\
\hline Janeiro/2015 & 99 & 10,6 & 33 \\
\hline Fevereiro/2015 & 119 & 12,8 & 39,7 \\
\hline Março/2015 & 134 & 14,4 & 44,7 \\
\hline Abril/2015 & 148 & 15,9 & 49,3 \\
\hline Total & 933 & 100 &
\end{tabular}

A classificação dos parâmetros analisados, posologia, via de administração, forma farmacêutica, medicamento inapropriado, inconsistências na dose prescrita e exames de glicemia desnecessários, evidenciam a ocorrências de notificações em todos os meses analisados. Nota-se que do total de 933 intervenções, 310, a maior quantidade foi referente à inconsistência na dose prescrita (Tabela 2).

Tabela 2. Quantidade de intervenções relacionadas às variáveis estudadas.

\begin{tabular}{lcc}
\hline Variáveis estudadas & $\mathrm{N}^{\circ}$ de intervenções & \% de intervenções \\
\hline Posologia & 263 & 28,2 \\
\hline Via de administração & 139 & 14,9 \\
\hline Forma farmacêutica & 47 & 5,0 \\
\hline Medicamento inapropriado & 116 & 12,4 \\
\hline Inconsistência na dose prescrita & 310 & 33,2 \\
\hline Exames de glicemia desnecessários & 58 & 6,3 \\
\hline
\end{tabular}

O mês em que mais ocorram intervenções relacionadas à posologia foi o mês de abril/2015, relacionadas à via de administração o mês de setembro/2014, relacionadas à forma farmacêutica fevereiro/2015, a dose janeiro/2015 e abril/2015, a medicamentos inapropriado janeiro/2015 e a exames de glicemia outubro/2014 (Tabela 3). 
Tabela 3. Quantidade de intervenções relacionadas às variáveis estudadas por mês.

\begin{tabular}{lcccccccccc}
\hline \multirow{2}{*}{ Variáveis } & \multicolumn{8}{c}{ Mês/ Ano } & \multirow{2}{*}{ Total } \\
\cline { 2 - 11 } & Set/14 & Out/14 & Nov/14 & Dez/14 & Jan/15 & Fev/15 & Mar/15 & Abr/15 & \\
\hline Posologia & 25 & 41 & 29 & 26 & 13 & 28 & 51 & 50 & 263 \\
\hline Via de administração & 20 & 16 & 16 & 17 & 15 & 17 & 19 & 19 & 139 \\
\hline Forma farmacêutica & 4 & 2 & 8 & 7 & 2 & 10 & 6 & 8 & 47 \\
\hline $\begin{array}{l}\text { Medicamento } \\
\text { inapropriado }\end{array}$ & 10 & 10 & 14 & 18 & 20 & 15 & 11 & 18 & 116 \\
\hline $\begin{array}{l}\text { Inconsistência na dose } \\
\text { prescrita }\end{array}$ & 29 & 35 & 39 & 38 & 44 & 41 & 40 & 44 & 310 \\
\hline $\begin{array}{l}\text { Exames de glicemia } \\
\text { desnecessários }\end{array}$ & 5 & 11 & 5 & 8 & 5 & 8 & 7 & 9 & 58 \\
\hline Total & 93 & 115 & 111 & 114 & 99 & 119 & 134 & 148 & 933 \\
\hline
\end{tabular}

\section{DISCUSSÃO}

Sabemos que médicos, farmacêuticos e enfermeiros estão amplamente envolvidos com o cuidado ao paciente. O farmacêutico clínico realiza suas intervenções junto ao médico e orienta a enfermagem sobre as particularidades de cada medicamento garantindo uma melhor eficácia no tratamento e contribuindo a não ocorrência de um evento adverso, evitando assim um dano maior ou irreversível ao paciente ${ }^{9}$. Observamos que antes do trabalho do farmacêutico clínico nestas unidades ocorriam erros de prescrições repetidamente e muitas vezes com um mesmo paciente, e após os trabalhos realizados pelos mesmos são raros os casos de um mesmo erro de prescrição vir a ocorrer novamente, mostrando o trabalho deste profissional.

Erros de prescrição resultam em danos ao paciente. Nota-se na Tabela 1 que em média 116 intervenções foram realizadas por mês, ou seja, 116 eventos adversos a medicamentos (EAM) foram evitados com a atuação do profissional farmacêutico. Um programa focado na revisão de prescrições medicamentosas possibilita a redução efetiva de erros de prescrição e danos relacionados ao uso medicamentos. Embora os médicos não estejam acostumados com a participação do farmacêutico, o número de intervenções aceitas mostra que as recomendações são clinicamente relevantes ${ }^{10,11}$.

Observa-se na Tabela 2 que o maior número de erros de prescrição são relacionados à posologia e a dose. A presença de medicamento inapropriado e erros relacionados à via de administração, também apresentaram prevalência elevada. Os resultados observados são semelhantes a resultados encontrados em outros estudos semelhantes ${ }^{8}$. Apropriadamente treinado, o farmacêutico clínico é especialista no uso de medicamentos, assim ele deve revisar e fazer recomendações, racionalizar a terapia medicamentosa com o objetivo de maximizar a segurança e os resultados. Esta atuação junto à equipe multidisciplinar reduz mortalidade, tempo de internação, diminuição do custo da terapêutica, efetividade e eficiência do tratamento ${ }^{9}$. Desse modo observa-se que ocorrem muitos problemas técnicos relacionados a medicamentos durante a rotina hospitalar, e o farmacêutico é o profissional de saúde mais capacitado a identificar e tomar medidas adequadas a fim de evitar que estes problemas prejudiquem de alguma forma a assistência fornecida $^{12,13}$.

Na Tabela 3, ressalta-se que o número de intervenções relacionadas à dose e a posologia aumentaram nos meses de março e abril, meses em que médicos recém formados entram para o programa de residência, e consequentemente devido a inexperiência os erros de prescrição se acentuam. O farmacêutico clínico deve estar integrado à equipe interdisciplinar, trabalhando lado a lado com os médicos, buscando contribuir com seus conhecimentos farmacológicos na qualidade do trabalho assistencial ${ }^{14}$.

A realização do presente estudo permitiu evidenciar a importância da inserção do profissional farmacêutico na equipe multiprofissional de assistência ao paciente devido aos benefícios das intervenções na terapêutica ocasionadas por sua presença. Sendo assim, diante dos resultados apresentados pôdese perceber que as intervenções farmacêuticas estão cada vez mais aceitas no contexto terapêutico e que o farmacêutico está também 
cada vez mais inserido na junta clínica. Estas intervenções trazem benefícios comprovados, tanto do ponto de vista clínico quanto do ponto de vista econômico, visto que leva ao uso racional dos medicamentos promovendo segurança ao paciente hospitalizado.

\section{CONFLITO DE INTERESSE}

Os autores declaram não haver qualquer potencial conflito de interesse que possa interferir na imparcialidade deste trabalho científico.

\section{REFERÊNCIAS}

1. Raduenz AC, Hoffmann P, Radunz V, Sasso GTMD, Maliska ICA, Marck PB. Cuidados de enfermagem e segurança do paciente: visualizando a organização, acondicionamento e distribuição de medicamentos com método de pesquisa fotográfico. Rev Latino-Am Enferm. 2010; 18(6): 1-10. DOI: http://dx.doi.org/10.1590/S010411692010000600002.

2. Capucho HC, Branquinho S, Reis LV. Gerenciamento de Risco e Segurança do Paciente. Ribeirão Preto: Cartilha Hospital das Clínicas da Faculdade de Medicina de Ribeirão Preto da Universidade de São Paulo; 2010.

3. Cardinal L, Fernandes C. Intervenção farmacêutica no processo da validação da prescrição médica. Rev Bras Farm Hosp Serv Saud. 2014; 5(2): 14-19.

4. Anacleto TA, Perini E, Rosa MB. Medication errors and drug-dispensing systems in a hospital pharmacy. Clinics. 2005; 60(4): 325-332. DOI: http://dx.doi.org/10.1590/S1807-

$\underline{59322005000400011}$.

5. Silva AEBC, Reis AMM, Miasso Al, Santos JO, Cassiani SHB. Eventos adversos a medicamentos em um hospital sentinela do Estado de Goiás, Brasil. Rev Latino-Am Enferm. 2011; 19(2): 1-9. DOI: $\quad$ http://dx.doi.org/10.1590/S010411692011000200021.

6. Benetoli A, Baron AF, Sangalli R, Bianchini OM, Bian VMC, Ferreira Neto CJB. Abreviaturas Perigosas e Descrições Inadequadas de Dose em prescrições de Medicamentos. Rev Bras Farm Hosp Serv Saud. 2011; 2(1): 10-14.

7. Nunes PHC, Pereira BMG, Nominato JCS, Albuquerque EM, Silva LFN, Castro IRS, et al. Intervenção Farmacêutica e Prevenção de Eventos Adversos. Rev Bras Cienc Farm 2008; 44(4): 692-699. DOI: http://dx.doi.org/10.1590/S1516$\underline{93322008000400016 .}$.

8. Reis WCT, Scopel CT, Correr CJ, Andrzejevski VMS. Análise das intervenções de farmacêuticos clínicos em um hospital de ensino terciário do Brasil. Einstein. 2013; 11(2): 190-196. DOI: http://dx.doi.org/10.1590/S1679$\underline{45082013000200010 .}$.

9. Pial R, Hegele V, Heineck I. Atuação do Farmacêutico Clínico em Unidade de Terapia Intensiva Adulto: Uma Revisão da Literatura. Rev Bras Farm Hosp Serv Saud. 2014; 5(1): 19-24.

10. Bond CA, Raehl CL, Franke T. Clinical pharmacy services, pharmacist staffing, and drug costs in United States hospitals. Pharmacotherapy. 1999; 19(12): 1354-1362. https://doi.org/10.1592/phco.19.18.1354.30893.

11. Van Den Bemt PM, Postma MJ, Van Roon EN, Chow MCC, Fijn R, Brouwers JRBJ. Cost-benefit analysis of the detection of prescription errors by hospital pharmacy staff. Drug Saf. 2002; 25(2): 135-143. https://doi.org/10.2165/00002018200225020-00006

12. Gimenes FRE, Marques TC, Teixeira TCA, Mota MLS, Silva AEBC, Cassiani SHB. Administração de medicamentos, em vias diferentes das prescritas, relacionada à prescrição médica. Rev Latino-Am Enferm. 2011; 19(1): 1-7.

13. Miranda TMM, Petriccione S, Ferracini FT, Borges Filho WM. Intervenções realizadas pelo farmacêutico clínico na unidade de primeiro atendimento. Einstein. 2012; 10(1): 74-78. DOI: http://dx.doi.org/10.1590/S1679$\underline{45082012000100015}$.

14. Martí MC, Torres NVJ. Manual para la atención farmacéutica. Valencia: Hospital Universitário Dr Peset; 2005. 
Recebido para publicação em 06/06/2016

Revisado em 19/06/2018

Aceito em 28/09/2018 